BULL. AUSTRAL. MATH. SOC.

VOL. I (1969), 81-108

\title{
Optimal quadrature
}

\section{S. Elhay}

Certain Hilbert spaces of functions with known smoothness are considered. The weights and mesh of the quadrature formulae which have least estimate of error with respect to the norm for functions in these spaces are found and their properties are discussed.

\section{Introduction}

In the problem of quadrature we are concerned with the approximation of the integral

$$
I(f)=\int_{t_{1}}^{t_{2}} f(x) d x
$$

by the sum

$$
Q_{n}(f)=\sum_{j=1}^{n} \sum_{i=1}^{N} A_{i j} f^{(i-1)}\left(x_{j}\right) .
$$

The accuracy of a quadrature depends on $n, A_{i j}$ and the $x_{j}$, and is measured in terms of the error

$$
E(f)=I(f)-Q_{n}(f) .
$$

Suppose that we are given a class $B$ of functions $f$ and that we characterize the accuracy of a quadrature formula for these functions by the real number

$$
\zeta=\sup _{f \in B}\left|I(f)-Q_{n}(f)\right|
$$

Received 21 March 1969. Received by J. Austral. Math. Soc. 15 July 1968. Communicated by G.B. Preston. 
The question that suggests itself is, can we for fixed $n$ select the $A_{i j}$ and $x_{j}$ so that they define a quadrature which minimizes this number $\zeta$ for the class $B$ ?

For an introduction to this type of quadrature with least estimate of the remainder, sometimes called "best" or "optimal" quadrature, see e.g. Krylov [2] (Chap. 8, p. 133) or Sard [3].

In [1] Kautsky characterizes this quadrature for the class of functions with bounded $L_{q}{ }^{(N)}$ norm and shows that the formula of order of precision $N-1$ involving the derivative of order $N-1$ does not use the $N-1$ st derivative if $N$ is even. In this paper we consider the Hilbert spaces ${ }_{N}$ in which the norm $\|f\|$ involves the function $f$, the derivative $f^{(N)}$ and may involve the derivatives $f^{(1)}$ up to $f^{(N-1)}$. Using the usual techniques of functional analysis we find some properties of the quadrature which minimizes $\zeta$ and explicitly derive the formulae for the cases $N=1,2$. Extending the results of [1] we show that for $N=2$ the optimal formula does not involve values of the derivatives $f^{t}(x)$ and we give the error bound $\zeta$.

\section{Definitions and notation}

(1) Let $P$ be the set of all partitions $p=\left(x_{1}, x_{2}, \ldots, x_{n}\right)$ which divide the finite interval $\left(t_{1}, t_{2}\right)$ into $n+1$ subintervals $I_{j}=\left(x_{j}, x_{j+1}\right)$ for $j=0, \ldots, n$ and where

$$
t_{1}=x_{0} \leqq x_{1}<x_{2}<\ldots<x_{n} \leqq x_{n+1}=t_{2}
$$

(2) Let $N$ be a positive integer and let $\alpha_{0}, \alpha_{1}, \alpha_{2}, \ldots, \alpha_{N}$ be a real sequence such that $\alpha_{0} \neq 0, \alpha_{N} \neq 0$. We shall then deal with the following Hilbert spaces:-

(i) For each fixed partition, $p \in P$, let $M_{N}$ be the space of real functions $f(x)$ such that the Nth derivative $f^{(N)}(x)$ has bounded norm in $L_{2}\left(t_{1}, t_{2}\right), f^{(N-1)}(x)$ is absolutely continuous on each subinterval $I_{j}$ of $\left(t_{1}, t_{2}\right)$, and the inner product is 


$$
(f, g)_{N}=\sum_{j=0}^{N} \alpha_{j}^{2} \int_{t_{1}}^{t_{2}} f^{(j)}(x) g^{(j)}(x) d x .
$$

(ii) Let $H_{N}$ be the subspace of $M_{N}$, independent of $p$, defined by

$$
\begin{aligned}
H_{N}= & \left\{f ; f \in M_{n}, \text { and } f^{(N-1)}(x)\right. \text { is absolutely } \\
& \text { continuous on the whole interval } \left.\left(t_{1}, t_{2}\right)\right\} .
\end{aligned}
$$

(3) We shall call $\tilde{Q}(n, M)$ the set of all $n$-point quadrature formulae $Q_{n}$ of the type (1.2) with the $A_{i j}$ real and the $x_{j}$ given by $p \in P$.

(4) For $p \in P$ we define $S_{p}^{N}$ as the collection of all error functionals of the type

$$
E(f)=I(f)-Q_{n}(f),
$$

where $Q_{n} \in \tilde{Q}(n, N)$ and has its mesh points $x_{j}$ given by $p$.

(5) Let $\tilde{Q}^{\prime}$ be a subset of $\tilde{Q}(n, N)$. The quadrature formula $Q_{n}^{*} \in \tilde{Q}^{\prime}$ will be said to be optimal in $\tilde{Q}^{\prime}$ over $H_{N}$ when $\left\|I-Q_{n}^{*}\right\| \leqq\left\|I-Q_{n}\right\|$ for all $Q_{n} \in \tilde{Q}^{\prime}$

(6) We will call the function $g$ defined on $\left(t_{1}, t_{2}\right)$ an exponential spline or e-splinel of degree $N$ with $n$ knots if for some partition $p \in P, g$ satisfies

$$
\sum_{j=0}^{N}\left(-j^{j} \alpha_{j}{ }^{2}{ }^{(2 j)}(x)=1 \text { for } x \in I_{i}, i=0, \ldots, n\right. \text {, }
$$

and

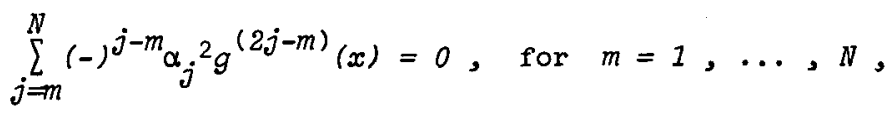

at $x=t_{1}$ if $x_{1} \neq t$, and at $x=t_{2}$ if $x_{n} \neq t_{2}$.

It is convenient to denote

1 The relation between best quadrature and polynomial spline functions [4] has been pointed out by Schoenberg [6] and more recently Karlin and Ziegler [7] have shown the relation between best quadrature and generalized splines [5]. 
(2.4) $g^{(k)}\left(x_{j}-\right)-g^{(k)}\left(x_{j}+\right)=R_{j k}, j=1, \ldots, n ; k=0, \ldots, 2 N-1$.

We will say the e-spline, $g$, has order of continuity $m$ when $R_{j k}=0$ for $j=1, \ldots, n$, and all $k=0,1, \ldots, m$. Let $G^{N}$ be the set of all e-splines of degree $N$ defined on the partition $p \in P$. We define

$$
G_{M}^{N}=\left\{g ; g \in G^{N} \text { and } g \text { has order } M\right\}
$$

Suppose for a moment that $t_{1} \neq x_{1}$ and $t_{2} \neq x_{n}$. In general the solution of $(2.2)$ depends on $2 N(n+1)$ constants called spline coefficients which are uniquely determined by the $2 N(n+1)$ conditions (2.3) and (2.4) for given $R_{j k}$. These conditions are linear equations with the spline coefficients as unknowns. To show that the spline is uniquely determined by this system we must show that the system of equations is non-singular. The conditions (2.3) and (2.4) with $R_{j k}=0$ for $j=1, \ldots, n$, and $k=0, \ldots, 2 N-1$, yield the corresponding system of homogeneous equations because the constant term $1 / \alpha_{0}{ }^{2}$, which is the particular integral of (2.2), vanishes by differentiation from (2.3) and (2.4), if $k \neq 0$, and cancels from (2.4) if $k=0$, by subtraction. Then it is sufficient to show that the trivial solution $g(x)=1 / \alpha_{0}{ }^{2}$, is the unique solution to this system.

Let $g^{*}$ be any solution of (2.2). By integrating

$$
\left(f, g^{*}\right)_{N}=\sum_{j=0}^{N} \alpha_{j}^{2} \int_{t_{1}}^{t_{2}} f^{(j)}(x) g^{*(j)}(x) d x, f \in H_{N}
$$

$N$ times by parts, and by setting the required conditions of the homogeneous system on $g^{*}$, we have

$$
\left(f, g^{*}\right)_{N}=I(f) \text {. }
$$

Directly expanding the inner product $(f, g)_{N}$ with $g=1 / \alpha_{0}{ }^{2}$ gives

$$
\left(f, 1 / \alpha_{0}^{2}\right)_{N}=I(f) \text {. }
$$

Now by the Hölder Inequality

$$
\left|\left(f, 1 / \alpha_{0}^{2}\right)\right| \leqq\left\|1 / \alpha_{0}^{2}\right\|\|f\|,
$$


or

$$
|I(f)| \leqq M\|f\|,
$$

where $M=\left\|1 / \alpha_{0}{ }^{2}\right\|$. Thus the functional $I(f)$ is bounded with respect to $\|f\|$ in $H_{N}$ and so by Riesz' theorem the function which realizes $I(f)$ is unique; but $R_{j k}=0$ implies that $g^{*} \in H_{2 N-1} \subset H_{N}$, so that

$$
g^{*}(x)=1 / \alpha_{0}^{2}
$$

Then the system is non-singular whenever $t_{1} \neq x_{1}$ and $t_{2} \neq x_{n}$. By similar argument when $t_{1}=x_{1}$ or $t_{2}=x_{n}$ the system can be seen to be nonsingular. Thus any spline of degree $N$ is uniquely determined by the jumps $R_{j k}$ for $j=1, \ldots, n$ and $k=0, \ldots, 2 N-1$.

The transformation to the new variable $h$ given by

$$
h= \begin{cases}x-\frac{x_{j}+x_{j+1}}{2}, & \text { for } x \in I_{j}, j=1, \ldots, n-1, \\ \frac{1}{2}\left(x-t_{1}\right)=\frac{1}{2}\left(x-x_{0}\right), & \text { for } x \in I_{0} \text { if } x_{0} \neq t_{1}, \\ \frac{1}{2}\left(x-t_{2}\right)=\frac{1}{2}\left(x-x_{n+1}\right), & \text { for } x \in I_{n} \text { if } x_{n} \neq t_{2},\end{cases}
$$

is useful as it transforms the subintervals, $I_{j}$, as follows

$$
\begin{aligned}
& I_{0}=\left(x_{0}, x_{1}\right) \rightarrow\left(0, \frac{h_{0}}{2}\right), \\
& I_{j}=\left(x_{j}, x_{j+1}\right) \rightarrow\left(-\frac{h_{j}}{2},+\frac{h_{j}}{2}\right), 1 \leqq j \leqq n-1, \\
& I_{n}=\left(x_{n}, x_{n+1}\right) \rightarrow\left(-\frac{h_{n}}{2}, 0\right), \text { where } h_{j}=x_{j+1}-x_{j} .
\end{aligned}
$$

We will consider e-splines to be functions of $x$ or $h$ as is necessary.

For convenience we shall denote

$$
Q_{n}(f)=\sum_{j=1}^{n} c_{j} f\left(x_{j}\right), \text { for the case } N=1 \text {, }
$$

and 


$$
Q_{n}(f)=\sum_{j=1}^{n} C_{j} f\left(x_{j}\right)+D_{j} f^{\prime}\left(x_{j}\right) \text {, for } N=2 \text {. }
$$

\section{Discussion and method}

(1) From the absolute continuity of the functions in $H_{N}$ and their derivatives of order $N-1$ it can be seen that $I(f)-Q_{n}(f)$ forms a linear and bounded functional over $H_{N}$. Then if we choose the set, $B$, as the subset of $H_{N}$ which contains functions with norm $\|f\|=1$, the definition (1.4) becomes

$$
\begin{aligned}
\zeta & =\sup _{f \in H_{N},\|f\|=1}\left|I(f)-Q_{n}(f)\right|, \\
& =\sup _{f \in H_{N},\|f\|=1}|E(f)|,
\end{aligned}
$$

and this supremum exists and is just $\|E\|_{H_{N}^{*}}$. Then

$$
|E(f)| \leqq\|E\|\|f\| \text {. }
$$

and so, by minimizing $\zeta$ we are reducing the size of the bound on $|E(f)|$ in the sense of the norm for functionals defined on ${ }_{N}$.

(2) In practical cases the choice of the metric for our set of functions and so in a sense the definition of $H_{N}$ will be left open. This choice should seek to give the best measure of the particular properties of interest of the functions in the space. For our case we have chosen an inner product which measures the smoothness of $f$ and its derivatives by integrating them in the square and summing their integrals. The integrals in the sum are weighted by the sequence of $\alpha_{j}$ 's so that for a particular problem this sequence can be chosen to minimize the product $\|E\|\|f\|$. The choice of these $\alpha_{j}{ }^{\prime} s$ is independent of the topology of $H_{N}$ and only affects our estimates of error.

In this paper we will consider only those sequences of $\alpha$ 's such that 
the polynomial,

$$
\sum_{j=0}^{N}(-)^{j} \alpha_{j}{ }^{2}{ }^{2 j}=0
$$

in $m$, which is the auxiliary equation to (2.2), has $2 N$ real and distinct roots

$$
\pm m_{1}, \pm m_{2}, \pm m_{3}, \ldots, \pm m_{N}
$$

In the case that the roots are multiple or complex the approach we have used, with slight modification, will lead to the corresponding optimal formula.

(3) For every bounded functional, $F$, defined on a Hilbert space, $H$, there exists a unique element, $g$, in that space which realizes $F$ by the relation

$$
(f, g)=F(f) \text { for any } f \in H \text {, }
$$

and

$$
\|g\|=\|F\| \text {. }
$$

Consequentiy for every error functional, $E$, on $H_{N}$ there is a function, $g$, which lies in ${ }_{N}$ and which satisfies (3.1) and (3.2). This relation between a Hilbert space and its dual enables us to find a particular element of the dual without dealing directly with the functionals. By finding the set of functions in $H_{N}$ which realize the errors, $E$, defined on some partition $p \in P$ and isolating that one, $\left(g^{*}\right)$, which has smallest norm we can construct the error which defines the optimal formula on that partition. This set is in fact $G_{N-1}^{N}$ and the following lemma summarises the relation between $G_{N-1}^{N}$ and the set, $S_{p}^{N}$, of error functionals on a given partition.

LEMMA 1. Suppose $p$ is a partition from $P$. There exists a one-to-one correspondence between the elements $E$ of $S_{p}^{N}$ and $g$ of $G_{N-1}^{N}$ such that

$$
(f, g)_{N}=E(f) \text { for any } f \in H_{N}
$$


Proof. (i) We note first that $G_{N-1}^{N} \subset H_{N}$. Let $g$ be from $G_{N-1}^{N}$ and $f$ from $H_{N}$.

$$
\begin{aligned}
(f, g)_{N} & =\sum_{i=0}^{N} \alpha_{i}{ }^{2} \int_{t_{1}}^{t_{2}} f^{(i)}(x) g^{(i)}(x) d x, \\
& =\sum_{j=0}^{n} \sum_{i=0}^{N} \alpha_{i}{ }^{2} \int_{I_{j}} f^{(i)}(x) g^{(i)}(x) d x .
\end{aligned}
$$

Integrating by parts $N$ times and using the conditions (2.2), (2.3) and

(3.4)

$$
R_{j k}=0, j=1, \ldots, n \text { and } k=0, \ldots, N-1 \text {, }
$$

gives

(3.5) $(f, g)_{N}=I(f)-\sum_{j=1}^{n} \sum_{i=0}^{N-1} f^{(i)}\left(x_{j}\right) \sum_{k=i+1}^{N}(-)^{k-i} \alpha_{k}{ }^{2} R_{j, 2 k-i-1}$.

Therefore if we choose

$$
A_{i j}=\sum_{k=i+1}^{N}(-)^{k-i} \alpha_{k}{ }^{2} R_{j, 2 k-i-1}
$$

for all $i=0, \ldots, N-1$, and $j=1, \ldots, n$, then we have

$$
\begin{aligned}
(f, g)_{N} & =I(f)-Q_{n}(f) \\
& =E(f) \text { as required. }
\end{aligned}
$$

(ii) Suppose now that $E$ is from $S_{p}^{N}$. With the $A_{i j}$ 's given by $E$ and $n N$ of the $R_{j k}$ given by (3.4), the relations (3.6) considered as a system of equations in the remaining $R_{j k}{ }^{\prime} s$ is in fact a triangular system. Then the $2 n N$ real numbers $R_{j k}$ defined by (3.4) and (3.6) uniquely define a spline $g \cdot$ By (3.4) $g$ belongs to $G_{N-1}^{N}$.

The elements of $S_{p}^{N}$ are bounded linear functionals and so by Riesz' theorem, $g$ is the unique element in $H_{N}$ which realizes $E$. This completes the proof of the lemma. 
Consider the set $G^{N}$ defined on the partition $p \in P$. All the elements of $G^{N}$ realize quadrature formulae and of the subcollection in $G^{N}$ which realize one quadrature formula, the single spline with smallest norm has a continuous derivative of order $N-1$. This subcollection is

$$
\begin{aligned}
J_{Q_{n}}^{N}= & \left\{g ; g \in G^{N} \text { such that for } Q_{n} \in \tilde{Q}(n, N)\right. \text { defined } \\
& \text { on } p \in P \text {, the } R_{j k} \text { satisfy }(3.6) \text { where the } A_{i j} \\
& \text { are given as the weights of } \left.Q_{n}\right\}
\end{aligned}
$$

and the continuity property of the spline, in ${ }_{Q_{n}}^{N}$, with smallest norm derives from the following lemma.

LEMMA 2. Let $g_{0} \in J_{Q_{n}}^{N}$ be such that $\left\|g_{0}\right\| M^{N} \leqq\|g\| M^{N}$ for any $g \in J_{Q_{n}}^{N}$. Then $g_{0} \in H_{N}$.

Proof. Since $J_{Q_{n}}^{N}$ is the set of all splines in $G^{N}$ which realize the the single quadrature formula $Q_{n}(f)$, the set $J_{Q_{n}}{ }^{N} \cap H_{N}$ contains only one element. This is because any function in this intersection will satisfy (3.6) from the definition of $J_{Q_{n}}^{N}$ and further it will have order of continuity $N-1$ because it belongs to $H_{N}$. Thus it will satisfy $R_{j k}=0$ for $j=1, \ldots, n$ and $k=0, \ldots, N-1$. As in the proof of Lemma 1, this uniquely defines a set of $2 n N$ jumps $R_{j k}$ which in turn define a single spline. Let us call this spline $g_{0}$. Then if $g$ is any element of $J_{Q_{n}}^{N}$ we may write

$$
g=g_{0}+Z
$$

But because $g$ and $g_{0}$ are both from $J_{Q_{n}}{ }^{N}$ they both realize the same quadrature formula, and thus the inner product $\left(g-g_{0}, f\right)$ vanishes for any $f \in H_{N}$. Then $(f, Z)=0$ for any $f \in H_{N}$ and $g_{0}$ is the projection of $g$ in $H_{N}$. By applying Pythagoras' theorem 


$$
\|g\|^{2}=\left\|g_{0}\right\|^{2}+\|z\|^{2} \text {, }
$$

we see that

$$
\left\|g_{0}\right\| \leq\|g\|
$$

for all $g \in J_{Q_{n}}^{N}$. Now let $g_{1} \in J_{Q_{n}}^{N}$ be such that $\left\|g_{1}\right\| \leqq\|g\|$ for any $g \in J_{Q_{n}}^{N}$. Then $\left\|g_{1}\right\|=\left\|g_{0}\right\|$. Further if we write $g_{1}$ as the sum

$$
g_{1}=g_{0}+2 \text {, }
$$

we see by relation (3.7) that $\|z\|=0$. Whence $\left\|g_{0}-g_{1}\right\|=0$ and so $g_{0}=g_{1}$ and the lemma is proved.

COROLLARY. Let $g^{*} \in G^{N}$ be such that $\left\|g^{*}\right\| M^{N} \leqq\|g\|_{M^{N}}$ for any $g \in G^{N}$. Then $g^{*} \in H_{N}$.

The proof follows immediately from the fact that

$$
G^{N}=\bigcup_{Q_{n}}{ }_{\tilde{Q}(n, N)} J_{Q_{n}}^{N}
$$

This result leads to an essential saving in the minimization process. It means that we can minimize $\|g\|$ over $G^{N}$ with respect to the spline coefficients independently on each subinterval and the resulting function will indeed have the properties of continuity which ensure that it lies in $G_{N-1}^{N}$. Using this technique we will find this spline $g^{*}$ and use it to construct the optimal quadrature formula for the mesh $p$.

It is interesting that for both of the cases $N=1$ and $N=2$ the spline with minimal norm has the property

$$
g^{*(k)}\left(x_{j}\right)=0, j=1, \ldots, n \text { and } k=0, \ldots, N-1 \text {, }
$$

independently of the partition $p$. This leads to the simple form

$$
\left\|E^{*}\right\|=\left\{\int_{t_{1}}^{t_{2}} g^{*}(x) d x\right\}^{\frac{1}{2}}
$$

for the error bound of the approximation. The result comes from the following lemma:- 
LEMMA 3. Suppose $g_{0} \in G_{N-1}^{N}$ has the property $g_{0}{ }^{(k)}\left(x_{j}\right)=0$ $j=1, \ldots, n$, and $k=0, \ldots, N-1$, then $\left\|g_{0}\right\| 2=I\left(g_{0}\right)$.

Proof. For all $f \in H_{N}, g \in G_{N-1}^{N}$, we have by (3.5)

$$
(f, g)_{N}=I(f)+\sum_{j=1}^{n} \sum_{i=0}^{N-1} f^{(i)}\left(x_{j}\right) \sum_{k=i+1}^{N}(-)^{k-i} \alpha_{k}^{2} R_{j, 2 k-i-1},
$$

Putting $f=g=g_{0}$ yields

$$
\begin{aligned}
\left(g_{0}, g_{0}\right) & =\left\|g_{0}\right\|^{2}=I(g)+\sum_{j=1}^{n} \sum_{i=0}^{N-1} g_{0}{ }^{(i)}\left(x_{j}\right) \sum_{k=i+1}^{N}(-)^{k-i} R_{j, 2 k-i-1}, \\
& =I\left(g_{0}\right),
\end{aligned}
$$

since $g_{0}{ }^{(i)}\left(x_{j}\right)=0$ for each $i$ and $j$.

This error bound will apply to any mesh distribution and, in particular, it will apply to the optimal mesh. Now $\left\|g^{*}\right\|$ is a function only of the mesh intervals $h_{0}, h_{1}, \ldots, h_{n}$. In order to find the optimal values of these intervals we must minimize $\left\|g^{*}\right\|$ with respect to $h_{0}, h_{1}, \ldots, h_{n}$. For the case $N=1$ this is done in one step but when $N=2$ we first consider $h_{0}$ and $h_{n}$ to be fixed and minimize $\left\|g^{*}\right\|$ with respect to $h_{1}, h_{2}, \ldots, h_{n-1}$, thereby getting as an intermediate result the optimal formula for the case that $h_{0}$ and $h_{n}$ are prescribed. The best values of $h_{0}$ and $h_{n}$ can then be found.

\section{The case $N=1$}

The elements of $G^{1}$ and $G_{0}^{l}$ defined on the partition $p \in P$ have the form

$$
g(x)=1 / a_{0}^{2}-\left(a_{j} \cosh r x+b_{j} \sinh r x\right),
$$

for all $x \in I_{j},(j=0,1, \ldots, n)$ where $r=\alpha_{0} / \alpha_{1}$ is the solution of the quadratic

$$
\alpha_{0}^{2}-\alpha_{1}^{2} m^{2}=0,
$$

and $a_{j}$ and $b_{j}$ are the spline coefficients. The results for the optimal 
formula on a fixed mesh and the description of the optimal mesh can be summarized as follows.

THEOREM I. (1) Let $\tilde{Q}_{p_{1}}$ be the subset of $\tilde{Q}(n, 1)$ containing all the quadrature formulae defined on the partition $p_{1} \in P$. The optimal quadrature formula $Q_{n}^{*}(f)$ in $\tilde{Q}_{p_{1}}$ over $H_{1}$ has its weights given by

$$
C_{j}=-\alpha_{1}^{2} R_{j 1}^{*}, \quad j=1, \ldots, n,
$$

where the e-spline $g^{*}$ is defined by

$$
a_{j}^{*}=\frac{1}{a_{0}{ }^{2} \cosh \frac{r h}{2} j}, \quad b_{j}^{*}=0, \quad j=0, \ldots, n,
$$

and satisfies

$$
g^{*}\left(x_{j}\right)=0, \quad j=1, \ldots, n .
$$

(2) The optimal formula in $\tilde{Q}(n, 1)$ has the weights $C_{j}$ as in (1) and has its mesh defined by

$$
h_{j}=\frac{t_{2}-t_{1}}{n}, \quad j=0, \ldots, n .
$$

Proof. (1) We shall minimize

$$
\|g\|^{2}=\sum_{j=0}^{n} \int_{I_{j}}\left(\alpha_{0}{ }^{2} g^{2}(x)+\alpha_{1}{ }^{2} g^{\prime 2}(x)\right) d x
$$

with respect to the spline coefficients $a_{j}$ and $b_{j}$. Denote

$$
M_{j}=\int_{I_{j}}\left(\alpha_{0}{ }^{2} g^{2}(x)+\alpha_{1}{ }^{2} g^{\prime 2}(x)\right) d x .
$$

In $1 \leqq j \leqq n-1$,

$$
M_{j}=\frac{b_{j}}{\alpha_{0}^{2}}+\frac{\alpha_{0}^{2}}{r}\left(a_{j}{ }^{2}+b_{j}{ }^{2}\right) \sinh r h_{j}-\frac{4 a_{j}}{r} \sinh \frac{r h_{j}}{2},
$$

is a quadratic function of $a_{j}, b_{j}$ which has a total differential of second order that is strictly positive whenever $h_{j}$ is positive. The normal equations

$$
a_{j}^{*} \frac{\alpha_{0}^{2}}{r} \sinh r h_{j}=\frac{2}{r} \sinh \frac{r h_{j}}{2},
$$


and

$$
b_{j}^{*} \frac{\alpha_{0}^{2}}{r} \sinh \frac{r h_{j}}{2}=0
$$

therefore define a minimum at the point

$$
a_{j}^{*}=\frac{1}{\alpha_{0}^{2} \cosh \frac{r h_{j}}{2}}, \quad b_{j}^{*}=0 \text {. }
$$

Now in the interval $I$ we have only one free spline coefficient because the end point condition (2.3) with $N=1$ has the form $g^{\prime}(0)=0$ implying $b_{0}^{*}=0$. Then

$$
M_{0}=\frac{h_{0}}{2 \alpha_{0}{ }^{2}}+\frac{\alpha_{0}{ }^{2}}{2 r} a_{0}^{2} \sinh r h_{0}-\frac{2 a_{0}}{r} \sinh \frac{r h_{0}}{2},
$$

and the normal equation

$$
a_{0}^{*} \frac{\alpha_{0}{ }^{2}}{r} \sinh r h_{0}=\frac{\sinh \frac{r h_{0}}{2}}{r},
$$

$$
\text { defines } a^{*}=\frac{1}{\alpha_{0}^{2} \cosh \frac{r h_{0}}{2}} \text {. }
$$

From the symmetry of the situation we can see that $a_{n}^{*}=a_{0}^{*}, b_{n}^{*}=-b_{0}^{*}$, and so

$$
g^{*}(h)=1 / \alpha_{0}{ }^{2}-\frac{\cosh r h}{\alpha_{0}{ }^{2} \cosh \frac{r h_{j}}{2}} .
$$

From this we have immediately

$$
g^{*}\left(x_{j}\right)=0, \quad j=1, \ldots, n
$$

(2) To find the optimal mesh distribution we use the method of the Lagrange multipliers. Let us construct a function

$$
\begin{aligned}
\omega & =\omega\left(h_{0}, h_{1}, \ldots, h_{n}\right), \\
& =\left\|g^{*}\right\|^{2}-\theta\left(\frac{h_{0}}{2}+\frac{h_{n}}{2}+\sum_{j=1}^{n-1} h_{j}-\left(t_{2}-t_{1}\right)\right),
\end{aligned}
$$


$\theta$ real. Recall that $g^{*}$ is expressed in terms of the mesh intervals $h_{0}, h_{1}, \ldots, h_{n}$. From the result (4.0) we may apply the Lemma 3 to $g^{*}$ and we arrive at

$$
\omega=\sum_{j=0}^{n} \int_{I_{j}} g^{*}(x) d x-\theta\left(\frac{h_{0}}{2}+\frac{h_{n}}{2}+\sum_{j=1}^{n-1} h_{j}-\left(t_{2}-t_{1}\right)\right) .
$$

From the form of $\omega$ over each interval $I_{j}$, setting $\frac{d w}{d h_{j}}=0$ for each $j$ defines a minimum for $\left\|g^{*}\right\|^{2}$ under the constraints

$$
\frac{h_{0}}{2}+\frac{h_{n}}{2}+\sum_{j=1}^{n-1} h_{j}=t_{2}-t_{1} \text {, for } h_{j} \geqq 0 \text {, each } j \text {. }
$$

By substituting for $a_{j}^{*}$ and $b_{j}^{*}$ in (4.1) and integrating, we see that

$$
\begin{aligned}
\omega & =\frac{t_{2}-t_{1}}{\alpha_{0}{ }^{2}}-\frac{1}{\alpha_{0}{ }^{2} r}\left(\tanh \frac{r h_{0}}{2}+\tanh \frac{r h_{n}}{2}+2 \sum_{j=1}^{n-1} \tanh \frac{r h_{j}}{2}\right) \\
& -\theta\left(\frac{h_{0}}{2}+\frac{h_{n}}{2}+\sum_{j=1}^{n-1} h_{j}-\left(t_{2}-t_{1}\right)\right) .
\end{aligned}
$$

Then $\frac{d w}{d h_{j}}=0$ implies

$$
\cosh ^{2} \frac{r h_{j}}{2}=\frac{-1}{\alpha_{0}{ }^{2} \theta} \text { for each } j=0, \ldots, n \text {. }
$$

But for each interval the equation for $h_{j}$ is independent of $j$ and, since all the equations have the same form, we may say that all the $h_{j}$ are equal and, by relation (4.2),

$$
h_{j}=\frac{t_{2}-t_{1}}{n}, \quad 0 \leqq j \leqq n .
$$

This completes the proof of Theorem I.

COROLLARY. The optimal formula in $\tilde{Q}(n, 1)$ over $H_{1}$ approaches the midpoint mile as the mesh size decreases.

Proof. For the optimal formula in $\tilde{Q}(n, 1)$ the weights are 


$$
\begin{aligned}
C_{j} & =-\alpha_{1}^{2} R_{j 1}^{*}, \\
& =-\alpha_{1}^{2}\left[g^{*^{\prime}}\left(+\frac{h_{j}}{2}-\right)-g^{*^{\prime}}\left(-\frac{h_{j}}{2}+\right)\right), \\
& =2 \frac{\alpha_{1}}{\alpha_{0}} \tanh \left(\frac{\alpha_{0}}{\alpha_{1}} \cdot \frac{t_{2}-t_{1}}{2 n}\right),
\end{aligned}
$$

which is a constant, $C$, since all the $h_{j}$ are equal. The midpoint rule

$$
Q_{n}^{T}(f)=\sum_{j=1}^{n} T_{j} f\left(x_{j}\right)
$$

has weights

$$
T_{j}=T=\frac{t_{2}-t_{1}}{n}
$$

and has the mesh points $x_{j}$ given by (4.3). If we denote

$$
B=\frac{t_{2}-t_{1}}{2} \cdot \frac{\alpha_{0}}{\alpha_{1}}
$$

then the ratio

$$
\frac{C}{T}=\frac{\tanh \frac{B}{n}}{\frac{B}{n}},
$$

is always less than unity, and

$$
\lim _{n \rightarrow \infty} \frac{C}{T}=1
$$

5. The case $N=2$

The elements of $G^{2}$ and $G_{1}^{2}$ defined on the partition $p \in P$ have the form

$$
g(x)=1 / \alpha_{0}^{2}-\left(a_{j} \cosh r x+b_{j} \cosh s x+c_{j} \sinh r x+d_{j} \sinh s x\right),
$$

for all $x \in I_{j}(j=0,1, \ldots, n)$ where the roots $\pm r, \pm s(r, s>0)$ of

$$
\alpha_{0}^{2}-\alpha_{1}{ }^{2} m^{2}+\alpha_{2}{ }^{2} m^{4}=0,
$$

are assumed to be distinct and real, and $a_{j}, b_{j}, c_{j}$ and $d_{j}$ are the spline coefficients. 
In each subinterval $I_{j}$ we denote

$$
C_{r}=\cosh r \omega_{j}, S_{r}=\sinh r \omega_{j},
$$

where $\omega_{j}=\frac{h_{j}}{2}, 0 \leqq j \leqq n-1$, and $\omega_{n}=-\frac{h_{n}}{2}$ and similarly for $c_{s}$ and $S_{s}$.

\subsection{The optimal formula for a fixed mesh}

THEOREM II. Let $p_{2}$ be a partition from $P$. Denote by $\tilde{Q}_{p_{2}}$ the subset of $\tilde{Q}(n, 2)$ containing all the quadrature formulae defined on the partition $p_{2}$. The optimal quadrature formula $Q_{n}^{*}(f)$ in $\tilde{Q}_{p_{2}}$ over $\mathrm{H}_{2}$ is given by the weights

$$
C_{j}=+\alpha_{2}^{2} R_{j 3}^{*}, D_{j}=-\alpha_{2}^{2} R_{j 2}^{*}
$$

where the spline $g^{*}$ is defined by

$$
\text { (1) } \begin{aligned}
a_{j}^{*} & =\frac{s S_{s}}{\alpha_{0}{ }^{2} T_{1}}, \quad b_{j}^{*}=\frac{-r S_{r}}{\alpha_{0}{ }^{2} T_{1}}, \\
T_{1} & =s C_{r^{S} S_{s}}-r S_{r} C_{s}, \\
c_{j}^{*} & =d_{j}^{*}=0, \quad(j=1, \ldots, n-1): \\
\text { (2) } \quad a_{j}^{*} & =\frac{r^{2} C_{r}-s^{2} C_{s}}{r \alpha_{0}{ }^{2} T_{2}}, b_{j}^{*}=-\frac{r^{2}}{s^{2}} a_{j}^{*}, \\
c_{j}^{*} & =\frac{-r\left(S_{r}-\frac{r}{s} S_{s}\right)}{\alpha_{0}{ }^{2} T_{2}}, d_{j}^{*}=-\frac{s}{r} c_{j},
\end{aligned}
$$

where

$$
T_{2}=\frac{1}{r}\left(C_{r}-\frac{r^{2}}{s^{2}} C_{s}\right)\left(r^{2} C_{r}-S^{2} C_{s}\right)-r\left(S_{r}-\frac{s}{r^{s}} S_{s}\right)\left(S_{r}-\frac{r}{s} S_{s}\right),(j=0 \text { and } n) \text {. }
$$

Further

$$
g^{*}\left(x_{j}\right)=g^{* \prime}\left(x_{j}\right)=0, j=1, \ldots, n .
$$

Proof. We will minimize

$$
\|g\|^{2}=\sum_{j=0}^{n} \int_{I_{j}}\left(\alpha_{0}{ }^{2} g^{2}(x)+\alpha_{1}{ }^{2} g^{\prime 2}(x)+\alpha_{2}{ }^{2} g^{\prime \prime 2}(x)\right) d x,
$$


with respect to the spline coefficients $a_{j}, b_{j}, c_{j}$ and $d_{j}$. Denote

$$
M_{j}=\int I_{j}\left(\alpha_{0}{ }^{2} g^{2}(x)+\alpha_{1}{ }^{2} g^{\prime 2}(x)+\alpha_{2}{ }^{2} g^{\prime \prime 2}(x)\right) d x \text {, }
$$

and consider first that $1 \leqq j \leqq n-1$. By expanding directly using the relations $r^{2} s^{2}=\frac{\alpha_{0}{ }^{2}}{\alpha_{2}{ }^{2}}$ and $r^{2}+s^{2}=\frac{\alpha_{1}{ }^{2}}{\alpha_{2}{ }^{2}}$, and integrating, we find that

$$
\begin{aligned}
M_{j}= & 2 \alpha_{1} 2\left\{r\left(a_{j}{ }^{2}+c_{j}{ }^{2}\right) S_{r} c_{r}+s\left(b_{j}{ }^{2}+a_{j}{ }^{2}\right) S_{s} C_{s}\right\} \\
& +4 \alpha_{0} \alpha_{2}\left\{\left(r a_{j} b_{j}+s c_{j} d_{j}\right) C_{r} S_{s}+\left(s a_{j} b_{j}+r c_{j} d_{j}\right) S_{r} c_{s}\right\} \\
& -4\left\{a_{j} \frac{S_{r}}{r}+b_{j} \frac{s}{r}\right\}+\frac{h_{j}}{\alpha_{0}^{2}} .
\end{aligned}
$$

As for the case $N=1, M_{j}$ is a quadratic function of $a_{j}, b_{j}, c_{j}$, and $d_{j}$ which has a total differential of second order that is strictly positive whenever $h$ is positive and the roots $r$ and $s$ are distinct and positive. Thus the extremal defined by the normal equations is a minimum. The system of normal equations

$$
\begin{aligned}
& r a_{j} \alpha_{1}^{2} S_{r} C_{r}+\alpha_{0} \alpha_{2} b_{j}\left(r C_{r} S_{s}+s S_{r} C_{s}\right)=\frac{S_{r}}{r}, \\
& s b_{j} \alpha_{1}^{2} S_{s} C_{s}+\alpha_{0} \alpha_{2} a_{j}\left(r C_{r} S_{s}+s S_{r} C_{s}\right)=\frac{S_{s}}{s}, \\
& r c_{j} \alpha_{1}{ }^{2} S_{r} C_{r}+\alpha_{0} \alpha_{2} d_{j}\left(s C_{r} S_{s}+r S_{r} C_{s}\right)=0, \\
& s d_{j} \alpha_{1}{ }^{2} S_{s} C_{s}+\alpha_{0} \alpha_{2} c_{j}\left(s C_{r} S_{s}+r S_{r} C_{s}\right)=0,
\end{aligned}
$$

has a non-vanishing determinant, so the unique solution is

$$
\alpha_{j}^{*}=\frac{s S_{s}}{\alpha_{0}{ }^{2} T_{1}}, \quad b_{j}^{*}=\frac{-r S_{r}}{\alpha_{0}{ }^{2} T_{1}}, \quad c_{j}^{*}=d_{j}^{*}=0,
$$

where

$$
T_{1}=\left(s C_{p} S_{s}-r S_{p} C_{s}\right)
$$

This means that, in the inside intervals, the spline defined by the normal equations is a symmetric function of its argument $h$. By substitution using (5.1) we have 


$$
\begin{aligned}
g^{* \prime}\left(\frac{h_{j}}{2}-\right) & =-\left\{r a_{j}^{*} S_{p}+s b_{j}^{*} S_{s}\right\}, \\
& =-\left\{\frac{\alpha_{1}^{2}}{r s}\left(s s_{r} C_{s}-r C_{p} s_{s}\right)-\alpha_{0} \alpha_{2} T_{1}\left[\frac{1}{r_{2}}+\frac{1}{s^{2}}\right]\right\}, \\
& =-\left\{\frac{\alpha_{1}^{2} \alpha_{2} T_{1}}{\alpha_{0}}-\frac{\alpha_{1}{ }^{2} \alpha_{2} T_{1}}{\alpha_{0}}\right\}, \\
& =0 .
\end{aligned}
$$

Similarly

$$
\begin{aligned}
g^{*}\left(\frac{h_{j}}{2}-\right) & =1 / \alpha_{0}{ }^{2}-\left(a_{j}^{*} C_{p}+b_{j}^{*} C_{s}\right) \\
& =\frac{\alpha_{0} \alpha_{2} T_{1}}{\alpha_{1}{ }^{2}}\left(\frac{b_{j}^{*}}{r S_{p}}+\frac{a_{j}^{*}}{s S_{s}}\right), \\
& =0 .
\end{aligned}
$$

Then from the symmetry of $g^{*}$ and the anti-symmetry of $g^{* \prime}$, it follows that

$$
g^{*}\left(x_{j}\right)=g^{* \prime}\left(x_{j}\right)=0, \quad \text { each } j=1, \ldots, n .
$$

This calculation verifies the continuity of $g^{*}$ and $g^{*}$ for the points $x_{2}, x_{3}, \ldots, x_{n-1}$ but, more than that, it gives an alternative system for the spline coefficients in the intervals $I_{0}$ and $I_{n}$. Of the four coefficients in $I_{0}$, two are determined by the end point condition (2.3) with $N=2$ and the other two may now be found explicitly from the two relations just derived. Clearly from the symmetry considerations the situation is the same for $I_{n}$. The system in $I_{0}$ and $I_{n}$ is, in fact,

$$
\left[\begin{array}{cccc}
C_{r} & c_{s} & s_{r} & s_{s} \\
r_{p} & s s_{s} & r C_{r} & s C_{s} \\
r^{2} & s^{2} & 0 & 0 \\
0 & 0 & s & r
\end{array}\right]\left[\begin{array}{c}
a_{j} \\
b_{j} \\
c_{j} \\
d_{j}
\end{array}\right]=\left[\begin{array}{c}
1 / \alpha_{0}{ }^{2} \\
0 \\
0 \\
0
\end{array}\right]
$$

for $j=0$ and $n$ and this system has solution 
$(5,3)$

$$
\begin{cases}a_{j}^{*}=\frac{r^{2} C_{p}-s^{2} C_{s}}{r \alpha_{0}{ }^{2} T_{2}}, & b_{j}^{*}=-\frac{r^{2}}{s^{2}} a_{j}^{*}, \\ c_{j}^{*}=-\frac{r\left(S_{r}-\frac{r}{s} S_{s}\right)}{\alpha_{0}{ }^{2} T_{2}}, & d_{j}^{*}=-\frac{s}{r} c_{j}^{*},\end{cases}
$$

where

$$
T_{2}=\frac{1}{r}\left(C_{r}-\frac{r^{2}}{s^{2}} C_{s}\right)\left(r^{2} C_{r}-s^{2} C_{s}\right)-r\left(S_{r}-\frac{s}{r} S_{s}\right)\left(S_{p}-\frac{r}{s} S_{s}\right)
$$

Now because $g^{*}$ is determined in $I_{0}$ and $I_{n}$ by the continuity conditions and normal equations for the intervals $I_{1}$ up to $I_{n-1}$, and since these normal equations define a minimum on each interval, we know that $g^{*}$ does indeed minimize $(5.0)$.

This completes the proof of Theorem II.

\subsection{The optimal mesh distribution}

We are now in a position to determine the distribution of mesh points which will minimize the error of approximation. In part (1) of Theorem III the mesh is described for the case that $h_{0}$ and $h_{n}$ are prescribed and in part (2) the system for the optimal values of $h_{0}$ and $h_{n}$ is given.

THEOREM III. The quadrature formula $Q_{n}^{*}(f)$ which is optimal in $\tilde{Q}(n, 2)$ over $H_{2}$ has its weights $C_{j}$ and $D_{j}$ given by Theorem II and its mesh given by

$$
\text { (1) } \begin{aligned}
h_{j} & =\frac{1}{(n-1)}\left[\left(t_{2}-t_{1}\right)-\left(\frac{h_{0}}{2}+\frac{h_{n}}{2}\right)\right], \\
& =\text { constant }, 1 \leqq j \leqq n-1 .
\end{aligned}
$$

(2) $h_{0}=h_{n}$, and $\int_{I_{0}} \frac{d}{d h_{0}} g^{*}(x) d x=\int_{I_{1}} \frac{d}{d h_{1}} g^{*}(x) d x$, where $g^{*}$ is the spline defined in Theorem II.

Proof. As for $N=1$ we define a function 


$$
\begin{aligned}
\omega & =\omega\left(h_{0}, h_{1}, \ldots, h_{n}\right) \\
& =\left\|g^{*}\right\|^{2}-\theta\left(\frac{h_{0}}{2}+\frac{h_{n}}{2}+\sum_{j=1}^{n-1} h_{j}-\left(t_{2}-t_{1}\right)\right), \theta \text { real. }
\end{aligned}
$$

In Theorem II it was shown that

$$
g^{*}\left(x_{j}\right)=g^{* \prime}\left(x_{j}\right)=0, \quad(j=1, \ldots, n),
$$

and, applying lemma 3 to $g^{*}$, we have

$$
\omega=\int_{t_{1}}^{t_{2}} g^{*}(x) d x-\theta\left(\frac{h_{0}}{2}+\frac{h_{n}}{2}+\sum_{j=1}^{n-1} h_{j}-\left(t_{2}-t_{1}\right)\right) .
$$

Once again from the form of $\omega$ over each subinterval $I_{j}$, the normal equations $\frac{d \omega}{d h_{j}}=0$ for each $j$ define a minimum point for $\left\|g^{*}\right\|^{2}$ under the constraints

$$
\frac{h_{0}}{2}+\frac{h_{n}}{2}+\sum_{j=1}^{n-1} h_{j}=t_{2}-t_{1}, \quad h_{j} \geq 0 .
$$

(1) Suppose first that $h_{0}$ and $h_{n}$ are prescribed. Then in $1 \leqq j \leqq n-1$ the normal equations are

$$
\frac{d}{d h_{j}} \int_{-\frac{1}{2} h_{j}}^{\frac{1 h}{2} j} g^{*}(x) d x=\theta
$$

Now $g^{*}$, for $x \in I_{j}$, is a function of the mesh interval $h_{j}$ only. Therefore all the equations (5.4) have exactly the same form and each one is independent of $j$ and we can say

$$
\begin{aligned}
& n_{j}=\frac{1}{(n-1)}\left[\left(t_{2}-t_{1}\right)-\frac{\left(h_{0}+h_{n}\right)}{2}\right], \\
= & \text { constant for all } j=1, \ldots, n-1 .
\end{aligned}
$$

(2) Suppose then that $h_{0}$ and $h_{n}$ are no longer fixed. We will write $g^{*}\left(h_{j} ; x\right)=g^{*}(x)$ to indicate that the coefficients of $g^{*}$ are functions of $h_{j}$ when $x \in I_{j}$. Setting $\frac{d \omega}{d h_{0}}=\frac{d \omega}{d h_{n}}=0$ yields 


$$
\frac{d}{d h_{0}} \int_{0}^{\frac{1}{2} h_{0}} g^{*}\left(h_{0} ; x\right) d x=\frac{d}{d h_{n}} \int_{-\frac{1}{2} h_{n}}^{0} g^{*}\left(-h_{n} ; x\right) d x=\frac{\theta}{2}
$$

Differentiating we get

$$
\begin{aligned}
& \frac{1}{2} g^{*}\left(h_{0} ; \frac{h_{0}}{2}\right)+\int_{0}^{\frac{1}{2} h_{0}} \frac{d}{d h_{0}} g^{*}(h ; x) d x \\
& =-\frac{1}{2} g^{*}\left(-h_{n} ;-\frac{h_{n}}{2}\right)+\int_{-\frac{1}{2} h_{n}}^{0} \frac{d}{d h_{n}} g^{*}\left(-h_{n} ; x\right) d x=\frac{\theta}{2} .
\end{aligned}
$$

But in $I_{0}$ and $I_{n}, a_{j}^{*}$ and $b_{j}^{*}$ are even functions of their arguments and $c_{j}^{*}$ and $d_{j}^{*}$ are odd functions. Then as

$$
g^{*}\left(h_{0} ; \frac{h_{0}}{2}\right)=g^{*}\left(-h_{n} ;-\frac{h_{n}}{2}\right)=0 \text {, }
$$

we may replace the above relation by

$$
\int_{0}^{\frac{1}{2} h_{0}} \frac{d}{d h} g^{*}\left(h_{0} ; x\right) d x=\int_{0}^{\frac{1}{2} h_{n}} \frac{d}{d h_{n}} g^{*}\left(h_{n} ; x\right) d x=\frac{\theta}{2},
$$

and, immediately,

$$
h_{0}=h_{n}
$$

Differentiating in (5.4), and noting again that $g^{*}\left( \pm \frac{h_{j}}{2}\right)=0$, we find that the relation

$$
\int_{0}^{\frac{1}{2} h_{0}} \frac{d}{d h_{0}} g^{*}\left(h_{0} ; x\right) d x=\int_{0}^{\frac{1}{2} h} \frac{d}{d h_{1}} g^{*}\left(h_{1} ; x\right) d x,
$$

which along with (5.5) and (5.6) gives us a complete characterization of the optimal mesh. This completes the proof of Theorem III.

\subsection{The weights to the derivative values}

COROLLARY 1. For the optimal formula in $\tilde{Q}(n, 2)$ over $H_{2}$ the derivative weights vanish, i.e. 


$$
D_{j}=0, \quad j=1, \ldots, n,
$$

and an alternative system for the mesh distribution is given by (5.5), (5.6) and $R_{12}^{*}=0$, where $g^{*}$ is the spline defined in Theorem II.

Proof. From relation (3.6) with $N=2$

$$
\begin{aligned}
D_{j} & =-\alpha_{2}^{2} R_{j 2}^{*}, \\
& =-\alpha_{2}^{2}\left(g^{* \prime}\left(x_{j}-\right)-g^{* \prime \prime}\left(x_{j}+\right)\right) .
\end{aligned}
$$

But in $1 \leqq j \leqq n-1$

$$
g^{* \prime \prime}\left(+\frac{h_{j}}{2}-\right)=g^{* \prime}\left(-\frac{h_{j}}{2}+\right)=-\left(r^{2} a_{j}^{*} C_{r}+S^{2} b_{j}^{*} C_{s}\right) \text {. }
$$

Substituting for $a_{j}^{*}$ and $b_{j}^{*}$ from (5.1) with all the $h_{j}$ equal, shows that $g^{* \prime}$ is continuous at all the points $x_{2}, \ldots, x_{n-1}$, whence $D_{2}=D_{3}=\ldots=D_{n-1}=0$. Because of symmetry it will suffice to show that $D_{1}=0$.

From Theorem II

$$
\begin{aligned}
& a c_{p}+b c_{s}=1 / a_{0}{ }^{2}, \\
& r a S_{p}+s b c_{s}=0
\end{aligned}
$$

where $a=a_{1}^{*}, b=b_{1}^{*}$ and, eliminating $b_{0}^{*}$ and $d_{0}^{*}$ by the conditions (2.3) with $N=2$,

$$
\begin{aligned}
& a_{0}\left(C_{r_{0}}-\frac{r^{2}}{s^{2}} C_{s_{0}}\right)+C_{0}\left(S_{r_{0}}-\frac{s}{r} S_{s_{0}}\right)=1 / \alpha_{0}^{2}, \\
& r a_{0}\left(S_{r_{0}}-\frac{r}{s} S_{s_{0}}\right)+\frac{C_{0}}{r}\left(r^{2} C_{r_{0}}-s^{2} C_{s_{0}}\right)=0,
\end{aligned}
$$

where $a_{0}=a_{0}^{*}, c_{0}=c_{0}^{*}$ and $c_{r_{0}}=\cosh \frac{r h_{0}}{2}$, etc. $((5.8)$ and $(5.9)$

result from $g^{*}\left(x_{1}+\right)=g^{* \prime}\left(x_{1}+\right)=0$ and $(5.10)$, (5.11) from $\left.g^{*}\left(x_{1}-\right)=g^{* \prime}\left(x_{1}-\right)=0\right)$. We will use the above equations to show that for the optimal values of $h_{0}$ and $h_{1}, R_{12}^{*}=0$.

The minimum condition (5.1) when integrated becomes 
Optimal quadrature

103

(5.12) $\frac{S_{r}}{r} \frac{d a}{d h_{1}}+\frac{S_{s}}{s} \frac{d b}{d h_{1}}=\frac{d a_{0}}{d h_{0}}\left(\frac{S_{r_{0}}}{r}-\frac{r^{2}}{s^{2}} S_{s_{0}}\right)+\frac{d c_{0}}{d h_{0}} \frac{\left(C_{r_{0}}-C_{s_{0}}\right)}{r}$.

If we differentiate (5.8) with respect to $h_{1}$ we get

$$
C_{r} \frac{d a}{d h_{1}}+C_{s} \frac{d b}{d h_{1}}+\frac{1}{2}\left(r a S_{r}+s b S_{s}\right)=0,
$$

and, since $g^{* \prime}\left(\frac{h_{1}}{2}\right)=0$,

$$
c_{r} \frac{d a}{d h_{1}}+c_{s} \frac{d b}{d h_{1}}=0 .
$$

Similarly, differentiating (5.9) with respect to $h_{1}$, we have

$$
\frac{1}{2}\left(r^{2} a c_{r}+s^{2} b c_{s}\right)+r \frac{d a}{d h_{1}} S_{r}+s \frac{d b}{d h_{1}} s_{s}=0,
$$

or

$$
r \frac{d a}{d h_{1}} S_{r}+s \frac{d b}{d h_{1}} S_{s}=\frac{1}{2} g^{* 1}\left(\frac{h_{1}}{2}\right) \text {. }
$$

Solving (5.13) and (5.14) for $\frac{d a}{d h_{1}}, \frac{d b}{d h_{1}}$ we find

$$
\frac{d a}{d h_{1}}=\frac{-1}{2 T_{1}} g^{* \prime}\left(\frac{h_{1}}{2}+\right) c_{s}, \frac{d b}{d h_{1}}=\frac{1}{2 T_{1}} g^{* \prime}\left(\frac{h_{1}}{2}+\right) c_{p} \text {, }
$$

where $T_{1}=s C_{r_{s}} S_{s}-r S_{r} C_{s}$.

By differentiating $(5.10)$ and (5.11) with respect to $h_{0}$ and solving the resulting system we get

$$
\begin{aligned}
& \frac{d a_{0}}{d h_{0}}=\frac{1}{2 T_{2}} g^{* 1}\left(\frac{h_{0}}{2}-\right)\left(S_{r_{0}}-\frac{s}{r} S_{s_{0}}\right), \\
& \frac{d b_{0}}{d h_{0}}=-\frac{1}{2 T_{2}} g^{* 1}\left(\frac{h_{0}}{2}-\right)\left(c_{r_{0}}-\frac{r^{2}}{s^{2}} c_{s_{0}}\right),
\end{aligned}
$$

where

$$
T_{2}=\left(C_{r_{0}}-\frac{r^{2}}{s^{2}} C_{s_{0}}\right) \frac{\left(r^{2} C_{r_{0}}-s^{2} C_{s_{0}}\right)}{r}-r\left(S_{r_{0}}-\frac{r}{s} S_{s_{0}}\right)\left(s_{r_{0}}-\frac{s}{r} S_{s_{0}}\right) .
$$

Equation (5.12) then becomes

https://doi.org/10.1017/S0004972700041307 Published online by Cambridge University Press 


$$
\begin{aligned}
& \text { (5.15) } \frac{g^{* \prime \prime}\left[\frac{h_{0}}{2}+\right]}{r s}\left[\frac{r C_{r} s_{s}-s S_{r} C_{s}}{s C_{r} s_{s}-r S_{r} C_{s}}\right]=\frac{g^{* n}\left(\frac{h_{0}}{2}-\right]}{T_{2}}\left[\left(C_{r_{0}}-\frac{r^{2}}{s^{2}} C_{s_{0}}\right) \frac{\left(C_{r_{0}}-C_{s_{0}}\right)}{r}\right. \\
& \left.-\left(S_{r_{0}}-\frac{s}{r} S_{s_{0}}\right]\left[\frac{s_{0}}{r}-\frac{r^{2}}{s^{3}} S_{s_{0}}\right)\right] \text {. }
\end{aligned}
$$

By elementary algebra it can be seen that

$$
\begin{aligned}
& \text { 16) }\left\{\left(C_{r_{0}}-\frac{r^{2}}{s^{2}} C_{s_{0}}\right) \frac{\left(C_{r_{0}}-C_{s_{0}}\right)}{r}-\left(s_{r_{0}}-\frac{s}{r} s_{s_{0}}\right)\left(\frac{S_{r_{0}}}{r}-\frac{r^{2}}{s^{3}} s_{s_{0}}\right)\right\} \\
& =\frac{1}{r^{2} s^{2}}\left\{r\left(C_{r_{0}}-C_{s_{0}}\right)\left(r^{2} C_{r_{0}}-s^{2} C_{s_{0}}\right)-\left(r^{3} S_{r_{0}}-s^{3} S_{s_{0}}\right)\left(s_{r_{0}}-\frac{r}{s} s_{s_{0}}\right)\right\},
\end{aligned}
$$

and so we can replace the term in square brackets on the right side of (5.15) by the whole of the right hand side of (5.16). But by substitution of the spline coefficients we see that

$$
g^{* \prime \prime}\left(-\frac{h_{1}}{2}+\right)=\frac{-r s}{\alpha_{0}{ }^{2}}\left[\frac{r C_{p} S_{s}-s S_{r} C_{s}}{s C_{p} S_{s}-r S_{r} C_{s}}\right],
$$

and

$$
\begin{aligned}
g^{* \prime \prime}\left(\frac{h_{0}}{2}-\right)= & \frac{-1}{\alpha_{0}^{2} T_{2}}\left[r\left(C_{r_{0}}-C_{s_{0}}\right)\left(r^{2} C_{r_{0}}-s^{2} C_{s_{0}}\right)\right. \\
& \left.-\left(r^{3} S_{r_{0}}-s^{3} S_{s_{0}}\right)\left(S_{r_{0}}-\frac{r}{s} S_{s_{0}}\right)\right],
\end{aligned}
$$

and so (5.15) becomes

$$
\left[g^{* \prime}\left(-\frac{h_{1}}{2}+\right)\right]^{2}=\left[g^{* \prime}\left(\frac{h_{0}}{2}-\right)\right]^{2},
$$

whence $R_{12}^{*}=0$.

\subsection{The optimal closed formula}

A quadrature formula in which the values of the function and its derivatives at the end points of the interval of integration are used is called a closed formula. The optimal $r_{\text {-point }}$ closed formula is easily obtained from Theorem III where it was shown that if $h_{0}$ and $h_{n}$ are 
prescribed, the remaining intervals $h_{1}, h_{2}, \ldots, h_{n-1}$ have equal length. The closed formula then is the case for which $h_{0}=h_{n}=0$.

COROLLARY 2. Let $\tilde{Q}_{p_{3}}$ be the subset of $\tilde{Q}(n, 2)$ containing those quadrature formulae whose partitions have $x_{1}=t_{1}$ and $x_{n}=t_{2}$. Then the quadrature formula $Q_{n}^{*}(f)$ which is optimal in $\tilde{Q}_{p_{3}}$ over $\mathrm{H}_{2}$ is characterized by

$$
\text { (1) } \begin{aligned}
2 C_{1} & =2 C_{n}=C_{j}=\frac{2\left(r^{2}-s^{2}\right) t_{p} t_{s}}{r s\left(r t_{p}-s t_{s}\right)}, j=2, \ldots, n-1, \\
\text { (2) } D_{j} & =0, j=2, \ldots, n-1, \\
D_{1} & =D_{n}=\frac{1}{r s}\left(\frac{r t_{s}-s t_{p}}{s t_{s}^{-r t}}\right),
\end{aligned}
$$

and

$$
\text { (3) } h_{j}=\frac{t_{2}-t_{1}}{n-1}, j=0, \ldots, n \text {, }
$$

where we have denoted

$$
t_{p}=\frac{S_{p}}{C_{p}} \text { and } t_{s}=\frac{S_{s}}{C_{s}} .
$$

Proof. From Theorem III part (1) we have, putting $h_{0}=h_{n}=0$,

As before

$$
h_{j}=\frac{t_{2}-t_{1}}{n-1}, \quad 1 \leqq j \leqq n-1 .
$$

As before

$$
D_{j}=0, \quad 2 \leqq j \leqq n-1 \text {, }
$$

and

$$
\begin{aligned}
c_{j} & =\alpha_{2}{ }^{2} R_{j 3}^{*}, \\
& =-2 \alpha_{2}{ }^{2}\left(r^{3} a_{j} s_{r}+s^{3} b_{j} S_{s}\right), 2 \leqq j \leqq n-1, \\
& =\frac{\left(r^{2}-s^{2}\right) t_{r} t_{s}}{r s\left(r t_{r}-s t_{s}\right)} .
\end{aligned}
$$

From Theorem II part (2) we can see that, since the spline coefficients in $I_{0}$ and $I_{n}$ are continuous functions of the mesh lengths $h_{0}$ and $h_{n}$, 


$$
\lim _{h_{0} \rightarrow 0} g^{* m}\left(\frac{h_{0}}{2}-\right)=\lim _{h_{n} \rightarrow 0}-g^{* \prime \prime}\left(-\frac{h_{n}}{2}+\right)=0,
$$

whence

$$
\begin{aligned}
C_{n}=C_{1} & =\alpha_{2}{ }^{2} R_{13}^{*}, \\
& =-\alpha_{2}{ }^{2} g^{*}\left(-\frac{h_{1}}{2}+\right), \\
& =-\alpha_{2}{ }^{2}\left(r^{3} a_{1} s_{r}+s^{3} b_{1} s_{s}\right), \\
& =\frac{1}{2} c_{j}, \quad 2 \leqq j \leqq n-1,
\end{aligned}
$$

since all the $h_{j}$ are equal.

Similarly

$$
\lim _{h_{0} \rightarrow 0} g^{* \prime \prime}\left(\frac{h_{0}}{2}-\right)=\lim _{h_{n \rightarrow 0}}-g^{* \prime \prime}\left(-\frac{h_{n}}{2}\right)=0 \text {, }
$$

and so

$$
\begin{aligned}
D_{n}=D_{1} & =-\alpha_{2}{ }^{2} g^{* \prime \prime}\left(-\frac{h_{n}}{2}+\right), \\
& =\alpha_{2}{ }^{2}\left(r^{2} a_{1} c_{r}+s^{2} b_{1} c_{s}\right), \\
& =\frac{1}{r s}\left(\frac{r s^{-s t}}{s t} s^{-r t}\right)
\end{aligned}
$$

\section{Concluding remarks}

For $N=1,2$ we have explicitly derived the weights and mesh which define the quadrature formula that has least estimate of error $|E(f)|$ with respect to the norm $\|f\|$ for functions in $H_{N}$. For $N=2$ we have obtained as intermediate results the optimal formulae when

(a) the mesh is prescribed, and

(b) only the two end intervals $h_{0}$ and $h_{n}$ of the mesh are prescribed.

The optimal closed formula has been found as a special case of (b) and two interesting points have emerged. 
Firstly, the optimal formula for $N=2$ does not involve the derivatives of the integrated function. This seems to indicate that there exists a generalization to higher $N$ which would in some way correspond to the results obtained in [1].

The second point of interest concerns the spline $g^{*}$ and its derivatives at the mesh points. The property

$$
\begin{array}{rl}
g^{*(k)}\left(x_{j}\right)=0 & j=1, \ldots, n \\
k & =0, \ldots, N-1
\end{array}
$$

is established for both cases $N=1$ and 2 . Together with the knowledge that the spline with minimal norm on a fixed partition is an even function in each interval $I_{1}, \ldots, I_{n-1}$, the conditions (6.1) completely define $g^{*}(x)$ and further they give a simple form for the multiplier $\left\|E^{*}\right\|$ in the error bound $\left\|E^{*}\right\|$. $\|f\|$ on $\left|E^{*}(f)\right|$. Thus while (6.1) is a necessary condition for the minimum it may also be a sufficient condition and a necessary and sufficent condition for larger values of $N$. Further results on these investigations will be made known as they become available.

This work was supported by a Control Data Australia Pty. Ltd. research scholarship at the Department of Computing Science, University of Adelaide, South Australia. I should also like to thank Professor J.A. Ovenstone and Dr J. Kautsky for their assistance and encouragement in this work.

\section{References}

[1] J. Kautsky, "Optimal quadrature formulae and minimal polynomials in $L_{q}^{(N)} "$, J. Austral. Math. Soc. (to appear).

[2] V.l. Krylov, Approximate calculation of integrals, (Macmillan, New York, 1962).

[3] Arthur Sard, "Best approximate integration formulas; best approximation formulas", Amer. J. Math. 71 (1949), 80-91.

[4] J.H. Ahlberg, E.N. Nilson and J.L. Walsh, Theory of splines and their applications (Academic Press, New York, 1967).

[5] J.H. Ahlberg, E.N. Nilson and J.L. Walsh, "Fundamental properties of 
generalized splines", Froc. Nat. Acad. Sci. U.S.A., 52 (1964), $1412-1419$.

[6] I.J. Schoenberg, "On monosplines of least deviation and best quadrature formulae", J. Soc. Indust. Appl. Math. Ser. B Numer. Anal. 2 (1965), 144-170.

[7] Samuel Karlin and Zoi Ziegler, "Chebyshevian spline functions", SIAM J. Numer. Anal. 3 (1966), 514-543.

Department of Computing Science,

The University of Adelaide. 